\title{
Quantitative Analysis of Progenitor Cell and Stem Cell Compartments in Normal versus Leukoplakia Affected Oral Mucosa- An Observational Study
}

\author{
Anjana Mazumdar ${ }^{1}$, Sandip Ghose², Avijit Hazra³ ${ }^{3}$ Debasish Pramanick ${ }^{4}$ \\ ${ }^{1}$ Department of Oral Pathology, Dr. R. Ahmed Dental College and Hospital, Kolkata, West Bengal, India. ${ }^{2}$ Department \\ of Oral Pathology, Dr. R. Ahmed Dental College and Hospital, Kolkata, West Bengal, India. ${ }^{3}$ Department of \\ Pharmacology, SSKM Hospital, Kolkata, West Bengal, India. ${ }^{4}$ Department of Oral Pathology, Dr. R. Ahmed Dental \\ College and Hospital, Kolkata, West Bengal, India.
}

\section{ABSTRACT}

\section{BACKGROUND}

Oral leukoplakia is a common potentially malignant disorder of the oral cavity which has the propensity to undergo malignant transformation over a period of time. Morphological alterations of the oral mucosa in the form of unscrappable white patch reflect the underlying cellular abnormalities. The available grading systems of epithelial dysplasia do not accurately predict which cases may eventually transform into malignancy.

\section{METHODS}

Here the characterization of stem cell and progenitor cell compartments has been done with the help of Cytokeratin 19 (K19) and c-Myc protein expression by Immunohistochemistry to evaluate the changes in normal non-keratinized oral mucosa and oral leukoplakia in population of Eastern India.

\section{RESULTS}

K19 expression is reduced in oral leukoplakia than normal oral mucosa with a mean value of $16.22 \pm 20.69$ (Standard Deviation). However, there is no statistically significant change in c-Myc expression with a mean value of $72.66 \pm 40.34$ (Standard Deviation).

\section{CONCLUSIONS}

The above study reveals that the expression of K19 is significantly decreased in oral leukoplakia compared to normal oral mucosa. c-Myc expression is showing lower value than in healthy oral mucosa but this difference is not significant statistically.

\section{KEY WORDS}

Oral Mucosa, Leukoplakia
Corresponding Author:

Debasish Pramanick,

Department of Oral Pathology,

Dr. R. Ahmed Dental College and Hospital,

114, AJC Bose Road, Kolkata,

West Bengal, India.

E-mail: dr.pramanick.debasish@gmail.com

DOI: $10.14260 /$ jemds/2019/774

Financial or Other Competing Interests: None.

How to Cite This Article:

Mazumdar A, Ghose S, Hazra A, et al. Quantitative analysis of progenitor cell and stem cell compartment in normal versus leukoplakia affected oral mucosa- an observational study. J. Evolution Med. Dent. Sci. 2019;8(48):3583-3587, DOI: $10.14260 /$ jemds $/ 2019 / 774$

Submission 04-10-2019,

Peer Review 13-11-2019,

Acceptance 19-11-2019,

Published 02-12-2019. 


\section{BACKGROUND}

Stem cell, a one of its kind cell type with unique capability of self-renewal and differentiation has long been under the scanner in scientific pursuit to determine the origin of cancer. Long back in the year 1997, the concept of tumor initiating stem cell in leukemia was published.(1) Since then, existence of these tumor initiating cells later on described as CSCs have been proved to be present in many other tumors like breast cancer,(2) brain tumor,(3) colon cancer,(4) pancreatic tumors ${ }^{(5)}$ and malignant melanoma.(6) Malignancy, a disease of high proliferation and turnover rate and with its other pathological aspects such as ability to metastasize, recur and its resistance to treatment are not understood completely. To explain this unknown biology behind malignancy, the concept of cancer stem cell has been posited. Even then controversy still persists, and many aspects of cancer biology cannot be explained by cancer stem cell theory.

Within a tumor, the neoplastic cells exhibit diverse phenotypic and functional features such as cell surface markers, genetic expression, differences in proliferation and invasion properties and therapeutic reactions. Considering these unique characteristics of cancer cells, two models have been proposed to explain the varied nature of the tumor cells. The stochastic model states that all tumor cells have the capability to undergo unlimited proliferation by undergoing genetic mutations depending on the intrinsic cues or stimulus. Those cells which undergo maximum changes lead to cancer development.(7) Another model, the cancer stem cell model postulates that there are some cells within the cancer which are quiescent and have stem cell like properties and a functional hierarchy exists within a tumor. These cells accumulate genetic mutations over a period of time due to external influences. The CSCs multiply as well as differentiate leading to cancer with heterogeneous population of cells. ${ }^{(8)}$

In an attempt to unify the 2 models, the clonal evolution theory was postulated. The clonal evolution theory has tried to reconcile the two models of cancer development. According to this theory, a normal cell undergoes changes in different stages of its division according to intrinsic cues to form precancerous stem cells (pCSCs). These cells further mutate during proliferation to form a mass of genetically varied cancer cells (CSCs). The CSCs constitute only a small population of cells within a tumor. The rest of the cancer cells have limited potential to proliferate and lack the potential to differentiate in multiple directions. The CSCs multiply as well as differentiate according to external cues leading to hierarchical genetic mutations and finally cancer with heterogeneous population of cells develops.(9)

Unlike many other malignancies, oral cancer is preceded by a preneoplastic lesion which is also found in case of cervical and intestinal cancer. To characterize the preneoplastic lesions more objectively the role of csc must be investigated further. Quantitative change of stem cells has already been demonstrated in precancerous lesions of the colon such as 'Familial adenomatous polyposis.'(10) One such preneoplastic lesion of the mouth is leukoplakia. Leukoplakia as defined by WHO in 2005 is a "white plaque of questionable risk having excluded other known diseases or disorders that carry no increased risk for cancer".

Global prevalence rate of oral leukoplakia in 2003 varied between $1.7 \%$ to $2.7 \%$ in general population with no difference between younger and older patients. The overall malignant progression of oral leukoplakia is about $5 \%$ or more. Thus, oral leukoplakia a clinically challenging precancerous lesion whose malignant transformation is difficult to predict. Currently there are no accepted biomarkers that can determine which lesions will progress to cancer. Stem cells logically have a role in its malignant transformation. (11),(12),(13),(14) As the stem cells at the basal layer and the transient amplifying cells belong to the proliferation unit of the epithelium, they undergo several cell cycles of division which make them prone to accumulate mutations leading to their genotypic changes which ultimately result in malignant transformation.(15),(16) So, quantification of stem cells and transient amplifying cells has a definite prognostic implication in determining the malignant transformation potentiality.

This study was carried out to quantify the stem cell population through expression of $\mathrm{K} 19$ in normal and dysplastic oral mucosa associated with oral leukoplakia as well as quantify the progenitor cell population through expression of c-Myc and to perform a comparative evaluation between the healthy and the diseased mucosa.

\section{METHODS}

Six biopsy specimens of normal buccal mucosa and twelve specimens of leukoplakia (Four each of Homogeneous, Speckled and Verrucous types) on buccal mucosa were taken for this study after written consent from the patients. The study was approved by the ethics committee of Dr. R. Ahmed Dental College and Hospital, West Bengal, India.

Tissues from oral leukoplakia located in buccal mucosa and tissues of normal buccal mucosa from patients with no history of tobacco and alcohol habits were included in the study. Tissues of leukoplakia associated with oral squamous cell carcinoma in buccal mucosa were excluded and patients with systemic diseases were also excluded from the study. The incisional biopsy samples were fixed in formalin for 24 hours and then processed for paraffin embedding for block preparation. 3 sections each 4 microns thick were made for H\&E staining and IHC staining. H\&E staining was done for confirmation of diagnosis and grading of dysplasia.

For immunostaining the sections were deparaffinised using EZ Prep Solution. Antigen retrieval was done by heat pretreatment in High pH Buffer (CC1) solution for 60 minutes. Endogenous peroxidase activity was blocked by Neu Vision blocking reagent for 4 minutes. Primary antibodies - c-Myc antibody (Biocare, reference \# CME415AK, CK, rabbit monoclonal, clone EP121, 1:100 dilution) and CK19 antibody (Lab vision, reference \# MS-198-PO, mouse monoclonal, clone A53-P/A2.26, 1:100 dilution) were used. Incubation time for both antibodies was 32 minutes at 37 degrees Centigrade. Secondary antibody used was Ultra-view detection system HRP multimer and incubation was done for 4 minutes. For chromogenic detection - UltraView Universal DAB Detection Kit (Ventana) was employed. Counterstaining was done by Gills Hematoxylin 8 minutes followed by Ventana Bluing Reagent 4 minutes. Slides were removed from stainer, cleared and mounted in DPX. 


\section{Statistical Analysis}

K19 and c-Myc expression values were averaged over 10 observation fields. Age and c-Myc mean values were normally distributed by Kolmogorov-Smirnov goodness-of-fit test, but K-19 mean values were skewed. Accordingly, the mean values were compared between oral leukoplakia group and normal (healthy control) group by Student's unpaired t test for c-Myc and Mann-Whitney U test for K19. Analysis was two-tailed and $\mathrm{p}$ value $<0.05$ was considered to indicate a statistically significant result. The linear association between K19 and cMyc expression was explored by constructing bivariate scatter plots and calculation of Spearman's rank correlation coefficient (rho) with its 95\% confidence interval. Statistica version 6 [Tulsa, Oklahoma: StatSoft Inc., 2001] and MedCalc version 11.6 [Mariakerke, Belgium: MedCalc Software 2011] software were used for statistical analysis.

\section{RESULTS}

Patients with oral leukoplakia of buccal mucosa ranging in age between 40 to 61 years of both sexes were selected for study and normal non keratinised buccal mucosa were taken as control from patients who were suffering from other lesions except leukoplakia and oral squamous cell carcinoma within the age group of 40 to 60 years. Table 1 shows Correlation between K19 \& CMyc expression in leukoplakia and Table 2 between Mean K19 \& CMyc Expression in Leukoplakia. Table 3 showing Confidence interval. Graph 01 Scatterplot shows Correlation between K19 \& CMyc in Normal Group and Graph 02- Scatterplot between Mean K19 \& CMyc in Control. Thus, the expression of $\mathrm{K} 19$ is significantly decreased in oral leukoplakia compared to normal oral mucosa; c-Myc expression is also showing lower value than in healthy oral mucosa but this difference is not significant statistically. In the oral leukoplakia group, linear correlation between K19 and cMyc expression was good with Spearman's rank correlation coefficient (rho) value of 0.592 ( $p=0.043 ; 95 \%$ confidence interval for Rho 0.027 to 0.870 ). In the normal oral mucosa controls, the correlation was however poor with Rho value of $-0.257(p=0.623 ; 95 \%$ confidence interval for Rho $0-0.884$ to 0.701 .

\begin{tabular}{|l|c|}
\hline Variable $Y$ & CMyc expression \\
\hline Variable X & K19 expression \\
\hline Table 1. Correlation between K19 Expression and CMyc Expression in \\
Oral Leukoplakia Group
\end{tabular}

\begin{tabular}{|c|c|}
\hline Sample Size & $\mathbf{1 2}$ \\
\hline Spearman's rank correlation coefficient (rho) & 0.592 (good) \\
\hline Significance level & $\mathrm{P}=0.043$ \\
\hline 95\% Confidence Interval for rho & 0.027 to 0.870 \\
\hline Table 2. Association between Mean K19 and Mean CMyc Expression in \\
Subjects with Oral Leukoplakia \\
\hline
\end{tabular}

\begin{tabular}{|c|c|}
\hline Sample size & 6 \\
\hline Spearman's rank correlation coefficient (rho) & -0.257 (poor) \\
\hline Significance level & $\mathrm{P}=0.623$ \\
\hline \multicolumn{2}{|c|}{$\begin{array}{c}\text { 95\% Confidence Interval for rho } \\
\text { Interval }\end{array}$} \\
\hline
\end{tabular}
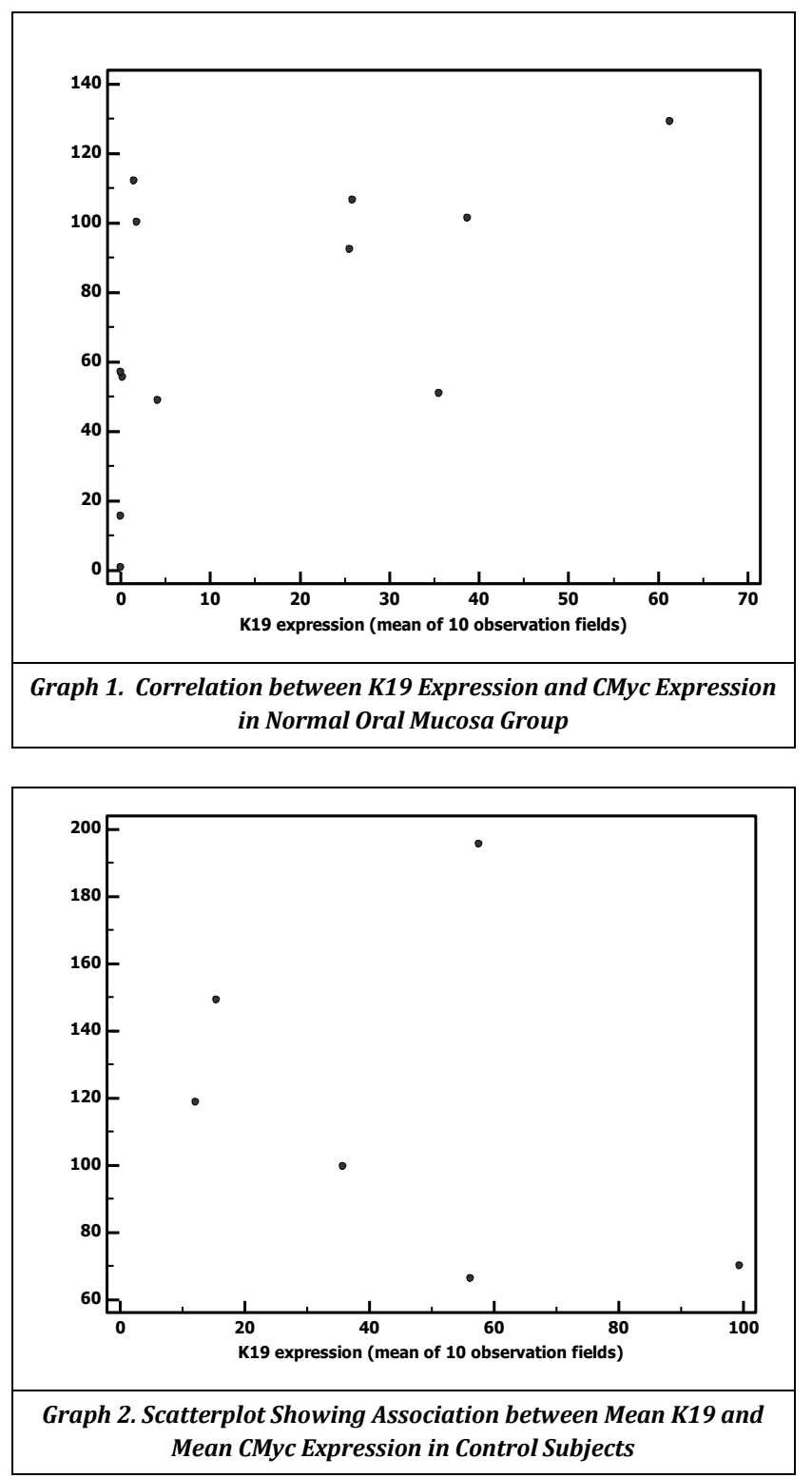

\section{DISCUSSION}

Oral leukoplakia is considered as one of the most common premalignant lesions. According to Mortazavi $\mathrm{H}$ et al in $2014,(17)$ the estimated prevalence rate of leukoplakia is $2 \%$ worldwide. The percentage of malignant transformation of oral leukoplakia is about $5 \%$ as reported by Parlatescu et al in 2014.(13) It is well known that the chances of malignant transformation increase with increasing severity of dysplasia and leukoplakia is often associated with varying degrees of dysplasia.(13) Though there is concurrence among oral pathologists about the oral epithelial dysplastic features, there is variation in interpreting severity of dysplasia. Till now there is no clinicopathological method by which it can be predicted accurately which clinical type of leukoplakia and its associated histological type will undergo malignant transformation. Many dysplastic lesions do not develop into cancer and some may even regress.

The WHO Collaborating Center for Oral Cancer and Precancer recommended the term 'Potentially malignant oral disorder' over 'Precancer' in order to emphasize that all 
disorders described would not transform to cancer.(12) As has been observed that even the clinically normal appearing mucosa in a patient harboring a precancerous lesion on the contra lateral anatomic site may have dysplasia or molecular aberrations in other oral mucosal sites suggestive of a pathway to malignant transformation and that cancer could subsequently arise in apparently normal tissue. Moreover, all precancerous lesions and conditions do not convert to malignancy. So, the term 'potentially malignant oral disorder' is preferred over the term 'precancer'. In search of accurate prediction of which leukoplakia will transform to malignancy several molecular markers have been tried and search for such a marker is continuing. So far none of the available molecular markers have demonstrated to be prognostically significant or have yet to be evaluated in large prospective studies.

Cancer is a disease of cell proliferation. So, attempt is made to explore the changes in the self-renewal and proliferative zone, of which stem cell is an essential part. According to Feller et al, the genetic mutations on normal stem cells and progenitor cells in the basal layer of oral epithelium leads to genetic instability in oral keratinocytes leading to development of functional precancerous phenotype.(18) These cells give rise to CSCs. These in turn can either form a monoclone of transformed cells or polyclones from multiple precursor cells. Ultimately there is clonal expansion and clonal divergence.

Characterization of changes in oral mucosa affected by leukoplakia can be done either by evaluation of protein expression or gene expression. Here, with the help of IHC, attempt has been made to explore the changes in expression of two important molecules related to stemness of the oral stem cells and in this respect the two markers that have been tried are K19,(19), (20),(21),(22) and c-Myc.(23),(24),(25) Though, the expression of K19 and c-Myc on oral dysplasia and oral cancer have been done earlier but, so far, no study has been carried out to look into the expression of these two proteins in the same tissue to characterize the basal stem cells and transient amplifying cells.

In this study it has been observed that K19 expression is reduced in oral leukoplakia compared to normal mucosa. This is in conformity with the findings of Su L, Morgan PR et al. They stated that in dysplasia, despite the presence of mRNA in keratinocytes, there is decrease in K19 protein expression. Here, RNA silencing may play a role which is still to be explored. K19 is a putative marker of stemness of keratinocytes which are slow cycling cells. The reduced expression of $\mathrm{K} 19$ in dysplasia explains the cellular proliferation along with the loss of stemness.

Another finding in this study is that unlike other epithelial tumors, the c-Myc expression remained unaltered. Previous studies on c-Myc showed increase in expression but in our study, it has been found to be unchanged. This maiden study has been conducted among patients from Eastern part of India. In this subpopulation this may be an important finding which contradicts the results coming out from other geographic regions. Further studies involving larger samples are warranted for confirmation of this result.

\section{CONCLUSIONS}

In this observational study, the expression of K19 and C-Myc has been explored to identify the stem cell and progenitor cell compartment of normal oral mucosa and oral leukoplakia. The K19 expression has been found to be decreased in leukoplakia in contrast to normal mucosa and C-Myc expression remained unaltered. However, larger studies are needed in Eastern India population to confirm the association between K19 or C-Myc expression in the oral mucosa and different clinical types of leukoplakia.

\section{REFERENCES}

[1] Bonnet D, Dick JE. Human acute myeloid leukemia is organised as a hierarchy that originates from primitive hematopoietic cell. Nature Medicine 1997;3(7):730-7.

[2] Al-Hajj M, Wicha MS, Benito-Hernandez A, et al. Prospective identification of tumorigenic breast cancer cells. Proc Natl Acad Sci U S A 2003;100(7):3983-8.

[3] Singh SK, Hawkins C, Clarke ID, et al. Identification of human brain tumor initiating cells. Nature 2004;432(7015):396-401.

[4] O'Brien CA, Pollett A, Gallinger S, et al. A human colon cancer cell capable of initiating tumour growth in immunodeficient mice. Nature 2007;445(7123):106-10.

[5] Li C, Heidt DG, Dalerba P, et al. Identification of pancreatic cancer stem cell. Cancer Res 2007;67(3):1030-7.

[6] Schatton T, Murphy GF, Frank NY, et al. Identification of cells initiating human melanomas. Nature 2008;451(7176):345-9.

[7] Odoux C, Fohrer H, Hoppo T, et al. A stochastic model for cancer stem cell origin in metastatic colon cancer. Cancer Res 2008;68(17):6932-41.

[8] Rich JN. Cancer stem cells: understanding tumor hierarchy and heterogeneity. Medicine (Baltimore) 2016;95(1 Suppl 1):S2-7.

[9] Song Y, Wang Y, Tong C, et al. A unified model of the hierarchical and stochastic theories of gastric cancer. British Journal of Cancer 2017;116(8):973-89.

[10] Ma H, Brosens LAA, Elias SG, et al. Longitudinal analysis of colon crypt stem cell dynamics in sulindac treated Familial Adenomatous Polyposis patients. Scientific Reports 2017;7(1):11972.

[11] Petti S. Pooled estimate of world leukoplakia prevalence: a systematic review. Oral Oncology 2003;39(8):770-80.

[12] Warnakulasuriya S, Johnson NW, Van der Waal I. Nomenclature and classification of potentially malignant disorders of the oral mucosa. J Oral Pathol Med 2007;36(10):575-80.

[13] Parlatescu I, Gheorghe C, Coculescu E, et al. Oral leukoplakia - an update. Maedica (Buchar): A Journal of Clinical Medicine 2014;9(1):88-93.

[14] Van der Waal I. Oral leukoplakia, the ongoing discussion on definition and terminology. Med Oral Patol Oral Cir Bucal 2015;20(6):e685-92. 14

[15] Blanpain C, Horsley V, Fuchs E. Epithelial stem cells: turning over new leaves. Cell 2007;128(3):445-58. 15 
[16] Gao JX. Cancer stem cells: the lessons from pre-cancerous stem cells. J Cell Mol Med 2008;12(1):67-96. 16

[17] Mortazavi H, Baharvand M, Mehdipour M, et al. Oral potentially malignant disorders: an overview of more than 20 entities. Journal of Dental Research, Dental Clinics, Dental Prospects 2014;8(1):6-14.17

[18] Fellar LL, Khammissa RR, Kramer BB, et al. Oral squamous cell carcinoma in relation to field pre-cancerisation: pathobiology. Cancer Cell International 2013;13(1):31.

[19] Lindberg K, Rheinwald JG. Suprabasal 40 kd keratin (K19) expression as an immunologic marker of pre-malignancy in oral epithelium. American Journal of Pathology 1989;134(1):89-98.

[20] Su L, Morgan PR, Thomas JA, et al. Expression of keratin 14 and 19 mRNA and protein in normal oral epithelia, hairy leukoplakia, tongue biting and white sponge nevus. J Oral Pathol Med 1993;22(4):183-9.

[21] Su L, Morgan PR, Lane EB, et al. Keratin 14 and 19 expression in normal, dysplastic and malignant oral epithelia. A study using in situ hybridisation and immunohistochemistry. J Oral Pathol Med 1996;25(6):293-301.

[22] Zong LP, Chen WT, Zhang CP, et al. Increased CK19 expression correlated with pathologic differentiation grade and prognosis in oral squamous cell carcinoma patients. Oral Surg Oral Med Oral Pathol Oral Radiol Endod 2007;104(3):377-84.

[23] Eversole LR, Sapp JP. c-myc oncoprotein expression in oral precancerous and early cancerous lesions. Eur J Cancer B Oral Oncol 1993;29B(2):131-5.

[24] Pai R, Pai S, Lalitha R, et al. Overexpression of c-Myc oncoprotein in oral squamous cell carcinoma in the South Indian population. Ecancermedicalscience 2009;3:128.

[25] Abdul-Majeed BA, Merza MS. Detection of transcription factor c-Myc in oral dysplasia and squamous cell carcinoma by in situ hybridization. J Fac Med Baghdad 2009;51(4):388-91. 